\title{
Effects of in-utero exposure to chemotherapy on fetal brain growth
}

Passera Sofia $^{1 *}$, Contarino Valeria ${ }^{2 *}$, Scarfone Giovanna ${ }^{2,3}$, Scola Elisa ${ }^{2}$, Fontana Camilla ${ }^{10^{* *}}$,

Peccatori Fedro ${ }^{4}$, Cinnante Claudia ${ }^{2}$, Counsell Serena ${ }^{5}$, Ossola Manuela ${ }^{2}$, Pisoni Silvia ${ }^{6}$, Pesenti Nicola $^{7}$, Grossi Elena ${ }^{2,3}$, Amant Frédéric ${ }^{8,9}$, Mosca Fabio ${ }^{10}$, Triulzi Fabio ${ }^{2,11}$, Fumagalli Monica ${ }^{2,10}$

* co-first authors; they have equally contributed

** corresponding author: camilla.fontana@mangiagalli.it, +39.3280342544. Via della commenda 12, Milano, Italy

${ }^{1}$ Clinica Mangiagalli, Milan, Italy

${ }^{2}$ Foundation IRCCS Ca’ Granda Ospedale Maggiore Policlinico, Milan, Italy

${ }^{3}$ University of Milan. Department of Obstetrics and Gynecology

${ }^{4}$ Fertility and Procreation Unit, Gynecologic Oncology Program, European Institute of Oncology, Milan, Italy.

${ }^{5}$ Centre for the Developing Brain, School of Bioengineering and Imaging Sciences, Kings College London, UK.

${ }^{6}$ Neonatology Unit, Mother and Child Department, Del Ponte Hospital, Azienda Socio Sanitaria Territoriale (ASST) Sette Laghi, Varese, Italy

${ }^{7}$ Department of Statistics and Quantitative Methods, Division of Biostatistics, Epidemiology and Public Health, University of Milano-Bicocca, Milan, Italy

${ }^{8}$ Center for Gynaecologic Oncology Amsterdam, Antoni van Leeuwenhoek-Netherlands Cancer Institute, and Amsterdam University Medical Centers, Netherlands

${ }^{9}$ Department of Oncology, KU Leuven, Belgium 
${ }^{10}$ University of Milan. Department of Clinical Sciences and Community Health

${ }^{11}$ University of Milan. Department of Pathophysiology and Transplantation

\section{Highlights:}

- Prenatal exposure to anthracycline/cyclophosphamide-based chemotherapy does not impact fetal brain growth.

- We provide evidence supporting the feasibility and safety of oncological treatment during pregnancy for the fetus.

- The safety of prenatal chemotherapy exposure on fetal brain growth may aid in discussions regarding time of delivery. 


\section{ABSTRACT}

\section{Objective}

Children exposed to chemotherapy in the prenatal period demonstrate normal neurocognitive development at 3 years but concerns regarding fetal brain growth remain high considering its vulnerability to external stimuli. Our aim was to evaluate the impact of in-utero chemotherapy exposure on brain growth and its effects on neurodevelopmental outcome.

Methods The protocol was approved by the local ethics committee. Brain regional volumes at term postmenstrual age were measured by magnetic resonance imaging (MRI) in children exposed to inutero chemotherapy and compared with normal MRI controls. Brain segmentation was performed by Advanced Normalization Tools (ANTs)-based transformations of the Neonatal Brain Atlas (ALBERT). Neurodevelopmental assessment (Bayley-III scales) was performed at 18 months corrected age in both exposed infants and in a group of healthy controls. Multiple linear regressions and false discovery rate correction for multiple comparisons were performed.

Results Twenty-one newborns prenatally exposed to chemotherapy (epirubicin administered in 80\% of mothers) were enrolled in the study: the mean gestational age (GA) was $36.4 \pm 2.4$ weeks and the mean birth weight was $2,753 \pm 622 \mathrm{~g}$. Brain MRI was performed at mean postmenstrual age of 41.1 \pm 1.4 weeks. No statistically significant differences were identified between the children exposed to chemotherapy and controls in both the total (398 $\pm 55 \mathrm{~cm}^{3}$ versus $427 \pm 56 \mathrm{~cm}^{3}$, respectively) and regional brain volumes. Exposed children showed normal Bayley-III scores (cognitive 110.2 \pm 14.5 , language $99.1 \pm 11.3$ and motor 102.6 \pm 7.3 ), and no significant correlation was identified between the brain volumes and neurodevelopmental outcome.

Conclusion Prenatal exposure to anthracycline/cyclophosphamide-based chemotherapy does not impact fetal brain growth, thus supporting the idea that oncological treatment in pregnant women seems to be feasible and safe for the fetus.

\section{INTRODUCTION}

Cancer is diagnosed in approximately 1 per 1000 pregnant women [1] and its incidence is increasing, as a result of a generalized tendency to conceive later in life [2]. The optimization of maternal treatment has led in recent years [1,3] to a decrease in the termination of pregnancy and iatrogenic-induced prematurity [1,4]. Chemotherapy represents a common treatment for the most frequent tumors diagnosed during pregnancy, namely breast cancer and lymphomas [1] and usually includes anthracyclines- and cyclophosphamide-based regimens [1]. The fetal effects of maternal drugs may occur and could depend on many factors, including the amount of drug that crosses the placenta and the capacity of the fetus to metabolize it $[5,6]$. Experimental evidence showed that less than $10 \%$ of doxorubicin and epirubicin reaches the fetal tissues [7]. In contrast, cyclophosphamide shows an easy penetration in the fetal compartment but the immature oxidative metabolism capacity of this drug in the fetus limits the conversion of the inactive parent compound into the active metabolite [7]. Despite these considerations, chemotherapy may exert detrimental impacts on the fetus through impairments in vascularization [1] and cellular growth of the placenta [8,9]. Moreover, 
considering the vulnerability of the developing central nervous system [10] throughout the whole gestation, chemotherapy may directly affect maturation and differentiation of cell types, as neurons [11] and oligodendrocytes [12], and it may have indirect neurotoxic effects related to oxidative stress $[13,14]$ and neuroinflammation [15]. Furthermore, the maternal illness, which may be associated with malnutrition, anemia and high maternal stress, could further negatively impact fetal development [1].

Data regarding short- and long-term neurodevelopmental outcomes in children prenatally exposed to chemotherapy seem reassuring when it is administered after the $14^{\text {th }}$ week of pregnancy, avoiding organogenesis [16]. The risk of congenital malformations is not increased regardless of the treatment for maternal disease and the drugs used [4], although most patients are treated with anthracyclines-based regimens in the second trimester and fetuses are not exposed to neurotoxic agents, such as methothrexate [17,18]. No consequence of cancer treatment was identified on neurobehavioral performances at 3 years, although an independent effect of prematurity on cognitive outcome was demonstrated [4]. Contradictory results have been published on the effects of chemotherapy on intrauterine growth but an increased rate of small for gestational age has recently been reported. [19]. Considering the vulnerability of the fetal brain to prenatal exposure to other toxic substances, such as drugs [20] or alcohol [21], and the absence of neuroimaging studies in newborn infants exposed in utero to chemotherapy, as well as, the known chemo-brain effect [2224], further knowledge on the potential detrimental effects of maternal cancer treatment on developing fetal brain is desirable. This is in order to tailor maternal therapy and identify infants at risk of impaired neurodevelopment. In this field, conventional MRI has been demonstrated to be an effective tool for detecting specific pattern of perinatal brain damage or abnormal brain development and predicting outcomes [25].

The aim of the present study is to assess the effect of prenatal exposure to chemotherapy on fetal brain growth in terms of volumetric development in a case-control setting. As a secondary aim, we explored the correlation between brain growth and infant neurobehavioral outcome at 18 months of age.

\section{METHODS}

We enrolled all infants born between June 2012 and December 2017 at the Fondazione IRCCS Ca' Granda, Ospedale Maggiore Policlinico of Milan, from mothers with any type of cancer diagnosed during pregnancy who were treated with any type of chemotherapy during gestation. Prenatally exposed infants were part of the Italian cohort of an international follow-up study by the International Network on Cancer, Infertility, and Pregnancy (INCIP) which is the biggest European network studying the effects of various cancer therapies on the health of both mother and child (https://www.cancerinpregnancy.org). At term postmenstrual, infants underwent brain MRI as part of the routine clinical care and after informed parental consent was signed MRI scans were retrospectively analyzed. According to the INCIP follow-up research protocol (approved by the local Ethics Committee), infants were assessed at 18 months of age using the Bayley Scales of infant neurodevelopment, third edition.

Prenatally exposed infants were matched with controls selected among infants born to healthy mothers at the same institution in the same years, after informed parental consent. 
Two different cohorts of control infants were selected:

1. MRI control group - Infants who had brain conventional MRI performed at term postmenstrual age for different clinical indications (prenatal or postnatal brain ultrasound abnormalities not confirmed at MRI; postnatal viral infections without any abnormalities at neurological examination and at MRI; family history of cerebral malformations without any evidence of brain malformations at MRI). Controls were matched in a 1:1 ratio for gestational age at birth and percentile of birth weight.

2. Neurodevelopment control group - Using the hospital electronic medical records control infants were randomly selected among a population of infants with the same gestational age at birth of the exposed infants; parents were contacted and voluntarily decided to allow their children to participate in this specific study as controls.

The following data were collected (refer to Supplementary Material-Table ST1 for details). Mother: age, trimester at diagnosis, type of cancer and type and dosage of chemotherapy, mode of delivery, twin pregnancy, causes of preterm delivery; infant: gestational age at birth, (categorized as late preterm $34^{+0}-36^{+6}$ weeks, moderate $32^{+0}-33^{+6}$ and very $<32$ ), birth weight and weight centile according to gestational age and sex, Apgar score; the neonatal perinatal morbidities: respiratory distress syndrome, retinopathy of prematurity, necrotizing enterocolitis, blood transfusion, jaundice, hypoglycemia, patent ductus arteriosus, intraventricular hemorrhage, periventricular leukomalacia, early and late-onset sepsis, and need for surgery; the neonatal data at neurological assessments: postmenstrual age (weeks) at brain MRI and age (months) at neurodevelopmental test.

\section{$\underline{\text { MRI scan assessment and volumetric analysis }}$}

A 3 T scanner (Achieva, Philips Healthcare, Best, The Netherlands) with pediatric-dedicated coil (Sense Ped, Philips Healthcare, Best, The Netherlands) was used. The brain MRI protocol included: 3D-T1 Fast Field Echo (FFE), T2 Turbo Spin Echo (TSE) coronal, T2 TSE axial, T2 FFE, and Diffusion-Weighted Imaging (DWI) (refer to Supplementary Material-Table ST2 for sequence parameters). The infants were scanned while sleeping and monitored by pulse oximetry and electrocardiography (Invivo Process monitoring; Invivo, Orlando, FL). MRI were assessed by a neuroradiologist blinded to the study group to evaluate the presence of brain injury, malformations and motion artifacts. The image processing pipeline of the volumetric analysis is presented in Figure 1 and detailed in Supplementary Material-Figure SF1.

\section{Neurodevelopmental assessment}

Neurodevelopment was assessed at 18 months (chronological age for term infants and corrected age for preterm infants), by a licensed examiner, using the Bayley Scales of Infant Development (third edition) which produces three composite scores: cognitive, language and motor. The scales have mean index scores of $100(\mathrm{SD} \pm 15)$ : higher scores indicate a more advanced development [26].

\section{Statistical analysis}

Demographic characteristics were reported as the mean (standard deviation - SD) or the number and percentage, as appropriate. Total brain volume (TBV) was estimated from the volumes of the single 
region of interest, ventricles excluded, and the relative volume of each region of interest was calculated as a fraction of the total brain volume. Independent t-test and linear regression were performed to investigate the differences in volume between the prenatally and not exposed groups, corrected for postmenstrual age at MRI. We subsequently performed false discovery rate correction for multiple comparisons. At the 18-month follow-up, differences in neurodevelopment between the groups, corrected for age at test, were assessed using multiple linear regression. The correlation between the total brain volume, corrected for the age at scan, and development at 18 months was calculated using partial correlation. Values of $\mathrm{p}<0.05$ were considered significant. Statistical analyses were performed using R version 3.4.4 (R Foundation for Statistical Computing, Vienna, Austria).

\section{RESULTS}

Thirty-one newborns were enrolled in the prenatally exposed group between June 2012 and December 2017 (Table 1-column A for maternal-neonatal characteristics and Supplementary material - Table ST3 for details on maternal treatment), of which 21 were included in the MRI volumetric analysis (Table 1-column B), 21 in the neurodevelopment assessment (Table 1-column D) and 15 in both. Each infant included in the analyses was matched with a control (Table 1-column $C$ for MRI control group and table 1-column E for neurodevelopment control group). The flow-chart of the recruitment is presented in Figure 2.

\section{Brain growth}

The 21 infants included in the MRI analysis were representative of the entire population of the prenatally exposed infants regarding both maternal and neonatal characteristic (Table 1-column $A-B$ ). The non-exposed infants were comparable to the prenatally exposed infants (Table 1-column $B-C)$. The mean postmenstrual age at the brain MRI was $41.1 \pm 1.4$ weeks and $42.8 \pm 1.9$ weeks in the prenatally exposed group and the non-exposed group; respectively $(\mathrm{p}=0.004)$. All infants had a normal neonatal neurological examination at term postmenstrual age. Neither congenital brain malformations nor acquired brain lesions were observed at MRI except for a typical prematurityrelated intraventricular hemorrhage-grade 1 with postnatal onset documented by cerebral ultrasound in two very preterm infants (one in each group). An effect of postmenstrual age on total brain volume at the MRI scan was observed (estimate $=22.9, \mathrm{p}<0.001, \mathrm{R}^{2}=0.552$ ), and the brain volume analysis was subsequently corrected for age at scan (Supplementary material-Figure SF2).

No significant difference in total brain volume between groups was observed, $398 \pm 55 \mathrm{~cm}^{3}$ and $427 \pm 56 \mathrm{~cm}^{3}$ in exposed and not exposed infants respectively, (IC 95\% -39;16; $\mathrm{p}=0.393$ ) (Table 2, Panel A) and either between the right and left side hemispheres (Table 2, Panel A). Similar results were observed after excluding the SGA infants $(\mathrm{p}=0.212)$ from the analysis or separately analyzing the term $(\mathrm{p}=0.177)$ or preterm $(\mathrm{p}=0.809)$ infants (Table 2, Panel B). The relative volumes of all 48 segmented areas were compared in the two groups: no significant differences were demonstrated even before the false discovery rate correction (Supplementary Material-Table ST4). No effect of side (left hemisphere versus right hemisphere) was identified. The total brain volume seemed to have no correlation with the cumulative dosage of epirubicin (estimate $=-0,02, \mathrm{p}=0.807$ ). 


\section{Neurodevelopmental outcome}

The 21 infants included in the neurodevelopmental analysis were representative of the entire population of the prenatally exposed infants regarding both maternal and neonatal characteristics (Table 1- column A-D). The not exposed infants were comparable to the prenatally exposed infants (Table 1-column D-E). All infants had a normal neonatal neurological examination at term postmenstrual age including the infants with intraventricular hemorrhage-grade 1. In the prenatally exposed group, the Bayley assessment was performed at a mean age of $19.8 \pm 3.2$ months, while in the not exposed group, it was performed at $18.8 \pm 2.5$ months $(\mathrm{p}=0.098)$. The cognitive $(110.2 \pm 14.5$ vs $111.4 \pm 13.8 ; p=0.787)$, language $(99.1 \pm 11.3$ vs $101.4 \pm 13.5 ; p=0.563)$ and motor $(102.6 \pm 7.3$ vs $103.2 \pm 11.7 ; \mathrm{p}=0.839)$ scores were not significantly different between the groups (Figure 3), even analyzing preterm and term infants separately (data not shown). The neurodevelopmental outcome at 18 months seemed to have no correlation with cumulative dosage of epirubicin (cognitive: estimate $=-0.005, \mathrm{p}=0.876$; language: estimate $=-0.017, \mathrm{p}=0.454$; and motor: estimate $=-0.004$, $\mathrm{p}=0.780)$. In the infants $(\mathrm{n}=15)$ who underwent both brain MRI and neurodevelopmental assessment, no relationship was observed between the total brain volume, and any of the subscales (cognitive: $0.183, \mathrm{p}=0.498$; motor: $0.309, \mathrm{p}=0.206$; and language: $0.205, \mathrm{p}=0.424$ ).

\section{DISCUSSION}

This study showed that fetal brain growth was not affected by maternal anthracyclines and cyclophosphamide-based chemotherapy; indeed infants prenatally exposed to chemotherapy and controls showed comparable total and regional brain volumes. The MRI study confirmed the absence of even subtle structural brain abnormalities supporting the safety of chemotherapy administered after the $1^{\text {st }}$ trimester of pregnancy in terms of the risk for congenital abnormalities. This result is a reassuring finding considering the potential harmful effect of chemotherapy administered during the $2^{\text {nd }}$ and $3^{\text {rd }}$ trimesters when fundamental stages of brain development (e.g. oligodendrogenesis and neurogenesis) occur $[11,12]$.

The absence of brain lesions, except for the typical prematurity-related intraventricular hemorrhage-grade 1 [27] developed postnatally by the preterm infant, suggests that maternal treatments during pregnancy do not seem to induce detrimental hemodynamic or metabolic perturbations in the fetus. Brain volumes were calculated from the MRI scan performed at term postmenstrual age and are consistent with previous findings [28,29]. Neonatal brain segmentation is a challenging task due to the motion, scan duration and rapid developmental brain changes. We used the validated ALBERT-ANTs segmentation method [30] which relies on a large atlas composed of manually segmented neonatal brains and the processing pipeline was adjusted to ensure robustness with MRI images acquired in clinical setting.

Consistently with previous findings, at 18 months of age, exposed infants showed neurodevelopmental scores within the normal ranges without significant differences between the groups [4]. In our population, we could not detect any differences in neurodevelopmental outcomes between preterm and full-term infants. However, the detrimental effect of prematurity on neurodevelopmental outcome is well documented [31], and our results may be affected by the small size of the two samples and cannot be generalized. Similarly, further studies are needed to confirm the absence of relationship between total brain volumes TBV and neurodevelopmental scores at 18 
months of age as this result was based on a limited population $(n=15)$. Although the rate of prematurity in the exposed children was relatively high (48\%) compared to the general population (approximately 10\%) [32], this finding is in line with previous data regarding infants prenatally exposed to chemotherapy (49\%) [1]. Moreover, most of these infants were born late preterm (80\%), and only 1 child was born before 32 weeks gestational age because of the poor clinical conditions of the mother; overall the rate of preterm delivery due to maternal reasons in our study was consistent with previous reports $(86 \%$ vs $88 \%)$ [1].

The incidence of small for gestational age infants was higher $(16 \%)$ than that in the general population (approximately 10\%) [33] but comparable to the incidence described for this specific group (21\%) [1]. These data are relevant when considering the increased risk for perinatal complications that affect small for gestational age infants [34]. Moreover, the clinical distinction between intra uterine growth restriction versus small for gestational age would be even more significant because the placental insufficiency underlying a fetal growth restriction in this population may be related to the malignancy, the surgery, the anesthesia, the chemotherapy and the general maternal conditions [1]. The majority of pregnant women in our population received anthracyclines and cyclophosphamide. These drugs are not considered neurotoxic, but anthracyclines have a wellknown dose-dependent cardiotoxicity. Only one patient with ovarian dysgerminoma received chemotherapy including cisplatin (instead of Cisplatin, etoposide, bleomycin (BEP)) and second line chemotherapy with paclitaxel and only one patient with lung cancer received one paclitaxelcarboplatin course. None of the prenatally exposed infants had cardiac abnormalities at the echocardiographic assessment in the first days after birth (results not shown) consistently with studies performed at 3 years of age [4].

The first limitation of our study is the small sample size; however, it reflects the low incidence of cancer in pregnancy. Based on this consideration, we used a robust magnetic resonance volumetric analysis applicable for standard quality images in clinical settings. Moreover, the limited spectrum of chemotherapy and the type of cancer analyzed in this study make our results not extendable to all infants prenatally exposed to chemotherapy. Nevertheless, considering that breast cancer is the tumor most diagnosed during pregnancy and that anthracyclines and cyclophosphamide are the most used drugs in women with cancer during pregnancy, our results may be useful for the counseling of women treated with these drugs during pregnancy.

In conclusion, we demonstrated that the brain of infants prenatally exposed to anthracycline/cyclophosphamide-based chemotherapy does not differ from controls in terms of the morphological appearance and volumetric development. These observations shed new light on the management of cancer during pregnancy, although caution is required when interpreting these findings. Our results strengthen the overall idea that oncological treatment in pregnant women is feasible and safe for the fetus, thus supporting and encouraging gynecologists and oncologists in handling this delicate and life-threatening condition for both mother and child. Further studies with larger sample sizes, advanced MRI methods, and longer follow-up will help further elucidate the microstructural development of the brain in these children and disentangle the potential effects of different chemotherapeutic agents, prematurity and fetal growth.

\section{Competing interests}


None declared

\section{Ethics Approval}

All infants included were part of the INCIP follow-up research protocol approved by the local Ethics Committee-Comitato Etico Milano Area B on the 15 of July 2014. In accord to the protocol, they were assessed at 18 months of age using the Bayley Scales of infant neurodevelopment, third edition. At term equivalent age, they underwent brain MRI as part of the routine clinical care, and MRI scans were retrospectively analyzed after informed parental consent was signed.

\section{Patient consent for publication}

Not required.

\section{Author contribution section}

SP participated to the design of the work and she managed the enrollment of patients (newborns) and the neonatal data collection; she gave substantial contribution to the analysis and interpretation of data. She coordinated the communication between the different participants. She wrote the first draft of the paper, she gave final approval of the version published and she ensured that questions related to the accuracy or integrity of any part of the work were appropriately investigated and resolved.

VC performed the volumetric analysis of the MRI scans. She wrote the first draft of the paper and she gave final approval of the version published ensuring that questions related to the accuracy or integrity of any part of the work were appropriately investigated and resolved.

GS participated in the enrollment of patients (mothers) at moment of cancer diagnosis and was involved in the management of maternal treatment. She gave substantial contributions to the analysis and interpretation of data, especially those regarding the maternal therapy. She revised the work with important intellectual content giving final approval of the version published.

ES performed and assessed the MRI scans. She gave technical support to Contarino V regarding the volumetric analysis. She gave substantial contributions to the analysis and interpretation of data, especially those regarding brain morphometry. She revised the work with important intellectual content giving final approval of the version published.

CF participated in enrollment of patients (newborns) and performed the neurodevelopment assessments. She gave substantial contributions to the analysis and interpretation of data, especially those regarding the follow up of the children. She revised the work with important intellectual content giving final approval of the version published.

FP contributed to the design of the clinical protocol. He participated in the management of maternal treatment. He gave substantial contributions to the analysis and interpretation of data, especially those regarding the maternal therapy. He revised the work with important intellectual content giving final approval of the version published.

CC performed and assessed the MRI scans. She revised the work with important intellectual content giving final approval of the version published. 
SC participated in finalizing the setting up of the tools to perform the volumetric analysis. She revised the work critically for important intellectual content and he gave final approval of the version published ensuring that questions related to the accuracy or integrity of any part of the work were appropriately investigated and resolved.

MO participated in the management of maternal treatment. She revised the work with important intellectual content giving final approval of the version published.

SP participated in enrollment of patients (newborns) and in the neonatal data collection. She revised the work with important intellectual content giving final approval of the version published.

NP performed the statistical analysis and gave a substantial contribution to the interpretation of data. He wrote and revised the work with important intellectual content and gave final approval of the version published.

EG participated in the management of maternal treatment. She revised the work with important intellectual content giving final approval of the version published.

FA revised the work critically for important intellectual content and he gave final approval of the version published ensuring that questions related to the accuracy or integrity of any part of the work were appropriately investigated and resolved.

FM gave a substantial contribution to the conception, design of the work, and interpretation of final data. He participated to the intellectual content revision of the work and he gave his final approval of the published version.

FT gave a substantial contribution to the conception, design of the work, and interpretation of final data. He participated to the intellectual content revision of the work and he gave his final approval of the published version.

MF was the project manager of the study. She coordinated and managed the whole project, contributing to the design of the clinical protocol. She gave substantial contribution to the acquisition, analysis and interpretation of data. She contributed to writing the paper and revised the work critically for important intellectual content and she gave final approval of the version published ensuring that questions related to the accuracy or integrity of any part of the work were appropriately investigated and resolved.

\section{Funding}

The authors have not declared a specific grant for this research from any funding agency in the public, commercial, or not-for-profit sectors.

\section{References}

1 Haan J De, Verheecke M, Calsteren K Van, et al. Oncological management and obstetric and neonatal outcomes for women diagnosed with cancer during pregnancy : a 20-year international cohort study of 1170 patients. 2018;19:337-46. doi: 10.1016/S1470- 
2045(18)30059-7.

2 Matthews TJ, Hamilton BE. Delayed childbearing: more women are having their first child later in life. NCHS Data Brief 2009;:1-8.http://www.ncbi.nlm.nih.gov/pubmed/19674536

3 Amant F, Halaska MJ, Fumagalli M, et al. Gynecologic cancers in pregnancy: Guidelines of a second international consensus meeting. Int J Gynecol Cancer 2014;24:394-403. doi:10.1097/IGC.0000000000000062

4 Amant F, Vandenbroucke T, Verheecke M, et al. Pediatric Outcome after Maternal Cancer Diagnosed during Pregnancy. N Engl J Med 2015;373:1824-34. doi:10.1056/NEJMoa1508913

5 Walker N, Filis P, Soffientini U, et al. Placental transporter localization and expression in the human: The importance of species, sex, and gestational age difference. Biol Reprod 2017;96:733-42. doi:10.1093/biolre/iox012

6 Han LW, Gao C, Mao Q. An update on expression and function of P-gp/ABCB1 and BCRP/ABCG2 in the placenta and fetus. Expert Opin Drug Metab Toxicol 2018;14:817-29. doi:10.1080/17425255.2018.1499726

7 Van Calsteren K. PhD Summary Chemotherapy during pregnancy : pharmacokinetics and impact on foetal neurological development. 2010;2:278-86. PMID: 25009715.

8 Cotechini T, Graham CH. Aberrant maternal inflammation as a cause of pregnancy complications: A potential therapeutic target? Placenta 2015;36:960-6.

doi:10.1016/j.placenta.2015.05.016

9 Verheecke M, Cortès Calabuig A, Finalet Ferreiro J, et al. Genetic and microscopic assessment of the human chemotherapy-exposed placenta reveals possible pathways contributive to fetal growth restriction. Placenta 2018;64:61-70.

doi:10.1016/j.placenta.2018.03.002

10 Cardonick E, Iacobucci A. Use of chemotherapy during human pregnancy. Lancet Oncol 2004;5:283-91. doi:10.1016/S1470-2045(04)01466-4

11 Haynes RL, Borenstein NS, Desilva TM, et al. Axonal development in the cerebral white matter of the human fetus and infant. J Comp Neurol 2005;484:156-67. doi:10.1002/cne.20453

12 Back SA, Luo NL, Borenstein NS, et al. Late oligodendrocyte progenitors coincide with the developmental window of vulnerability for human perinatal white matter injury. $J$ Neurosci 2001;21:1302-12. PMID:11160401

13 Oboh G, Ogunruku OO. Cyclophosphamide-induced oxidative stress in brain: Protective effect of hot short pepper (Capsicum frutescens L. var. abbreviatum). Exp Toxicol Pathol 2010;62:227-33. doi:10.1016/j.etp.2009.03.011

14 Doğan Z, Kocahan S, Erdemli E, et al. Effect of chemotherapy exposure prior to pregnancy on fetal brain tissue and the potential protective role of quercetin. Cytotechnology 2014;67:1031-8. doi:10.1007/s10616-014-9742-z

15 Vercruysse DC-M, Deprez S, Sunaert S, et al. Effects of prenatal exposure to cancer 
treatment on neurocognitive development, a review. Neurotoxicology 2016;54:11-21. doi:10.1016/j.neuro.2016.02.013

16 Amant F, Calsteren K Van, Halaska MJ, et al. Long-term cognitive and cardiac outcomes after prenatal exposure to chemotherapy in children aged 18 months or older : an observational study. 2012;13. doi:10.1016/S1470-2045(11)70363-1

17 Cardonick E, Iacobucci A. Use of chemotherapy during human pregnancy. Lancet Oncol 2004;5:283-91. doi:10.1016/S1470-2045(04)01466-4

18 Van Calsteren K, Heyns L, De Smet F, et al. Cancer During Pregnancy: An Analysis of 215 Patients Emphasizing the Obstetrical and the Neonatal Outcomes. J Clin Oncol 2010;28:683-9. doi:10.1200/JCO.2009.23.2801

19 Peccatori FA, Fumagalli M. Long and Winding Road of Cancer and Pregnancy: A Need for Action. J Clin Oncol 2017;35:1499-500. doi:10.1200/JCO.2017.72.4856

20 Warton FL, Meintjes EM, Warton CMR, et al. Prenatal methamphetamine exposure is associated with reduced subcortical volumes in neonates. Neurotoxicol Teratol 2018;65:519. doi:10.1016/j.ntt.2017.10.005

21 Riley EP, Mcgee CL. SYMPOSIUM Fetal Alcohol Spectrum Disorders: An Overview with Emphasis on Changes in Brain and Behavior. Experiemental Biol Med 2005;230:358-65. doi:10.1177/15353702-0323006-03

22 Inagaki M, Yoshikawa E, Matsuoka Y, et al. Smaller regional volumes of brain gray and white matter demonstrated in breat cancer survivors exposed to adjuvant chemotherapy. Cancer 2007;109:146-56. doi:10.1002/cncr.22368

23 Koppelmans V, De Ruiter MB, Van Der Lijn F, et al. Global and focal brain volume in longterm breast cancer survivors exposed to adjuvant chemotherapy. Breast Cancer Res Treat 2012;132:1099-106. doi:10.1007/s10549-011-1888-1

24 Reddick WE, Shan ZY, Glass JO, et al. Smaller white-matter volumes are associated with larger deficits in attention and learning among long-term survivors of acute lymphoblastic leukemia. Cancer 2006;106:941-9. doi:10.1002/cncr.21679

25 Manuel Hinojosa-RodríguezT. H, C. C-P, J.D. VH, et al. Clinical neuroimaging in the preterm infant: Diagnosis and prognosis. NeuroImage Clin 2017;16:355-68. doi:http://dx.doi.org/10.1016/j.nicl.2017.08.015

26 Albers CA, Grieve AJ. Test Review: Bayley, N. (2006). Bayley Scales of Infant and Toddler Development- Third Edition. San Antonio, TX: Harcourt Assessment. J Psychoeduc Assess 2007;25:180-90. doi:10.1177/0734282906297199

27 Ballabh P. Intraventricular Hemorrhage in Premature Infants: Mechanism of Disease. Pediatr Res. 2010 January ; 67(1): 1-8. doi:10.1203/PDR.0b013e3181c1b176.

28 Gousias IS, Hammers A, Counsell SJ, et al. Magnetic Resonance Imaging of the Newborn Brain: Automatic Segmentation of Brain Images into 50 Anatomical Regions. PLoS One 2013;8:e59990. doi:10.1371/journal.pone.0059990

29 Alexander B, Kelly CE, Adamson C, et al. Changes in neonatal regional brain volume 
associated with preterm birth and perinatal factors. Neuroimage Published Online First: 21 July 2018. doi:10.1016/j.neuroimage.2018.07.021

30 Gousias IS, Edwards AD, Rutherford MA, et al. Magnetic resonance imaging of the newborn brain: Manual segmentation of labelled atlases in term-born and preterm infants. Neuroimage 2012;62:1499-509. doi:10.1016/j.neuroimage.2012.05.083

31 Aarnoudse-Moens CSH, Weisglas-Kuperus N, van Goudoever JB, et al. Meta-Analysis of Neurobehavioral Outcomes in Very Preterm and/or Very Low Birth Weight Children. Pediatrics 2009;124:717-28. doi:10.1542/peds.2008-2816

32 Blencowe $\mathrm{H}$, Cousens $\mathrm{S}$, Chou D, et al. Born too soon: the global epidemiology of 15 million preterm births. Reprod Health 2013;10 Suppl 1:S2. doi:10.1186/1742-4755-10-S1-S2

33 Metcalfe A, Lisonkova S, Joseph K. The association between temporal changes in the use of obstetrical intervention and small- for-gestational age live births. BMC Pregnancy Childbirth 2015;15:233. doi:10.1186/s12884-015-0670-5

34 Tsai LY, Chen YL, Tsou KI, et al. The impact of small-for-gestational-age on neonatal outcome among very-low-birth-weight infants. Pediatr Neonatol 2015;56:101-7. doi:10.1016/j.pedneo.2014.07.007 


\section{Table 1}

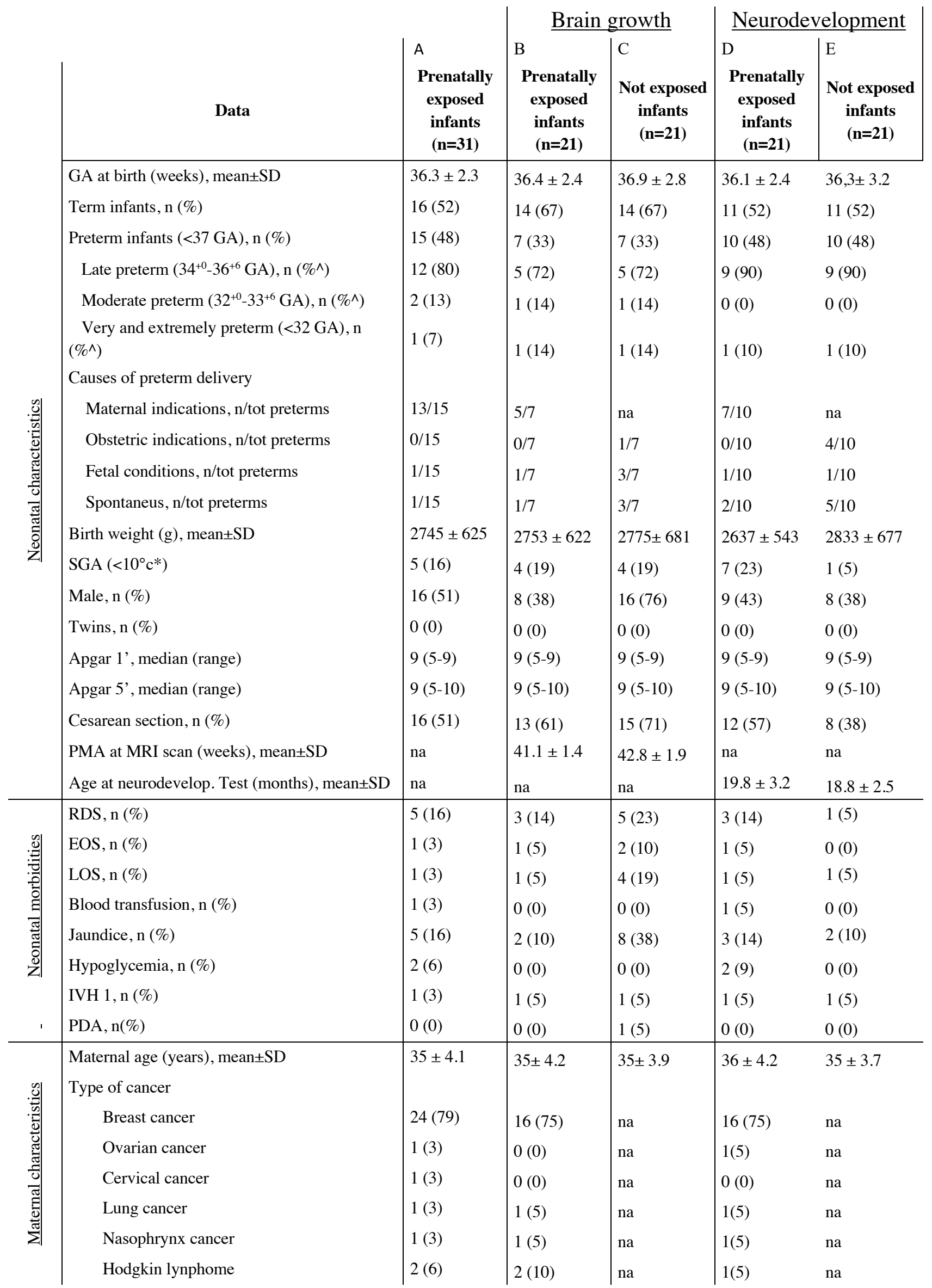




\begin{tabular}{|l|l|l|l|ll}
\multicolumn{1}{|c|}{ Non Hodgkin lynohome } & $1(3)$ & $1(5)$ & na & $1(5)$ & na \\
Diagnosis in the 2nd trimester, n (\%) & $21(67)$ & $11(52)$ & na & $12(57)$ & na \\
Patients exposed to epirubicin, n (\%) & $26(83)$ & $17(81)$ & na & $18(85)$ & na \\
Cum. dos. epirubicin (mg/m2), median (range) & $252(75-450)$ & $280(75-450)$ & na & $262(75-450)$ & na
\end{tabular}

Table 1. Maternal and neonatal characteristics of the study groups. Only observed neonatal morbidities are reported. Column A: all prenatally exposed group included in the study; Column B: prenatally exposed infants whose MRI was analyzed; Column C not exposed infants who performed MRI; Column D: prenatally exposed infants who performed neurodevelopmental test at 18 months; Column E not exposed infants who performed neurodevelopmental test at 18 months. Maternal and neonatal data of 10/31 prenatally exposed infants were previously published [4]. *according to Fenton (for preterm infants) and the WHO (for term infants) growth chart. GA: gestational age, PMA: post menstrual age, SGA: small for gestational age, MRI: magnetic resonance imaging, RDS: respiratory distress syndrome, EOS: early onset sepsis, LOS: late onset sepsis, IVH: intraventricular hemorrhage, PDA: patent ductus arteriosus, na: not applicable; $\left(\%{ }^{\wedge}\right)$ respect to total number of preterm infant; Maternal indications: severe maternal clinical conditions and/or planning maternal treatment; fetal conditions: e.g. intrauterine growth restriction, fetal ascites; obstetric indication: cholestasis, gestational hypertension, placenta praevia; spontaneous: e.g. premature rupture of membranes, beginning of labor

\section{Table 2}

\begin{tabular}{|c|c|c|c|c|c|c|c|c|}
\hline \multicolumn{9}{|l|}{$\underline{\text { Panel A }}$} \\
\hline \multirow[b]{2}{*}{ Volumes } & \multicolumn{3}{|c|}{ Prenatally exposed infants } & \multicolumn{3}{|c|}{$\underline{\text { Not exposed infants }}$} & \multirow[b]{2}{*}{$95 \%$ CI } & \multirow[b]{2}{*}{ p.value } \\
\hline & Number & $\operatorname{Mean}\left(\mathrm{cm}^{3}\right)$ & $\mathrm{SD}\left(\mathrm{cm}^{3}\right)$ & Number & Mean $\left(\mathrm{cm}^{3}\right)$ & $\mathrm{SD}\left(\mathrm{cm}^{3}\right)$ & & \\
\hline Total volume & 21 & 398 & 55 & 21 & 427 & 56 & {$[-39 ; 16]$} & 0.393 \\
\hline Left volume & 21 & 184 & 26 & 21 & 196 & 26 & {$[-19 ; 7]$} & 0.326 \\
\hline Right volume & 21 & 185 & 26 & 21 & 200 & 26 & {$[-17 ; 9]$} & 0.524 \\
\hline
\end{tabular}

$\underline{\text { Panel B }}$

\begin{tabular}{|c|c|c|c|c|c|c|c|c|}
\hline \multirow[b]{2}{*}{ Volumes } & \multicolumn{3}{|c|}{ Prenatally exposed infants } & \multicolumn{3}{|c|}{$\underline{\text { Not exposed infants }}$} & \multirow[b]{2}{*}{$95 \% \mathrm{CI}$} & \multirow[b]{2}{*}{ p.value } \\
\hline & Number & Mean $\left(\mathrm{cm}^{3}\right)$ & $\mathrm{SD}\left(\mathrm{cm}^{3}\right)$ & Number & $\operatorname{Mean}\left(\mathrm{cm}^{3}\right)$ & $\operatorname{SD}\left(\mathrm{cm}^{3}\right)$ & & \\
\hline Total volume - AGA & 17 & 405 & 59 & 17 & 420 & 55 & {$[-48 ; 11]$} & 0.212 \\
\hline $\begin{array}{l}\text { Total volume } \\
\text { Preterm }\end{array}$ & 7 & 420 & 55 & 7 & 414 & 67 & {$[-52 ; 41]$} & 0.809 \\
\hline Total volume - Term & 14 & 387 & 54 & 14 & 433 & 51 & {$[-71 ; 14]$} & 0.177 \\
\hline
\end{tabular}

Table 2. Total brain volumes. Comparison between prenatally exposed and not exposed infants in the whole population (Panel A) and AGA (adequate for gestational age), preterm and term analyzed separately (Panel B). Comparisons obtained using multiple linear regression, corrected for post menstrual age at brain MRI. The results are expressed as the mean $\pm \mathrm{SD}$. 


\section{Figures}

\section{Figure 1}

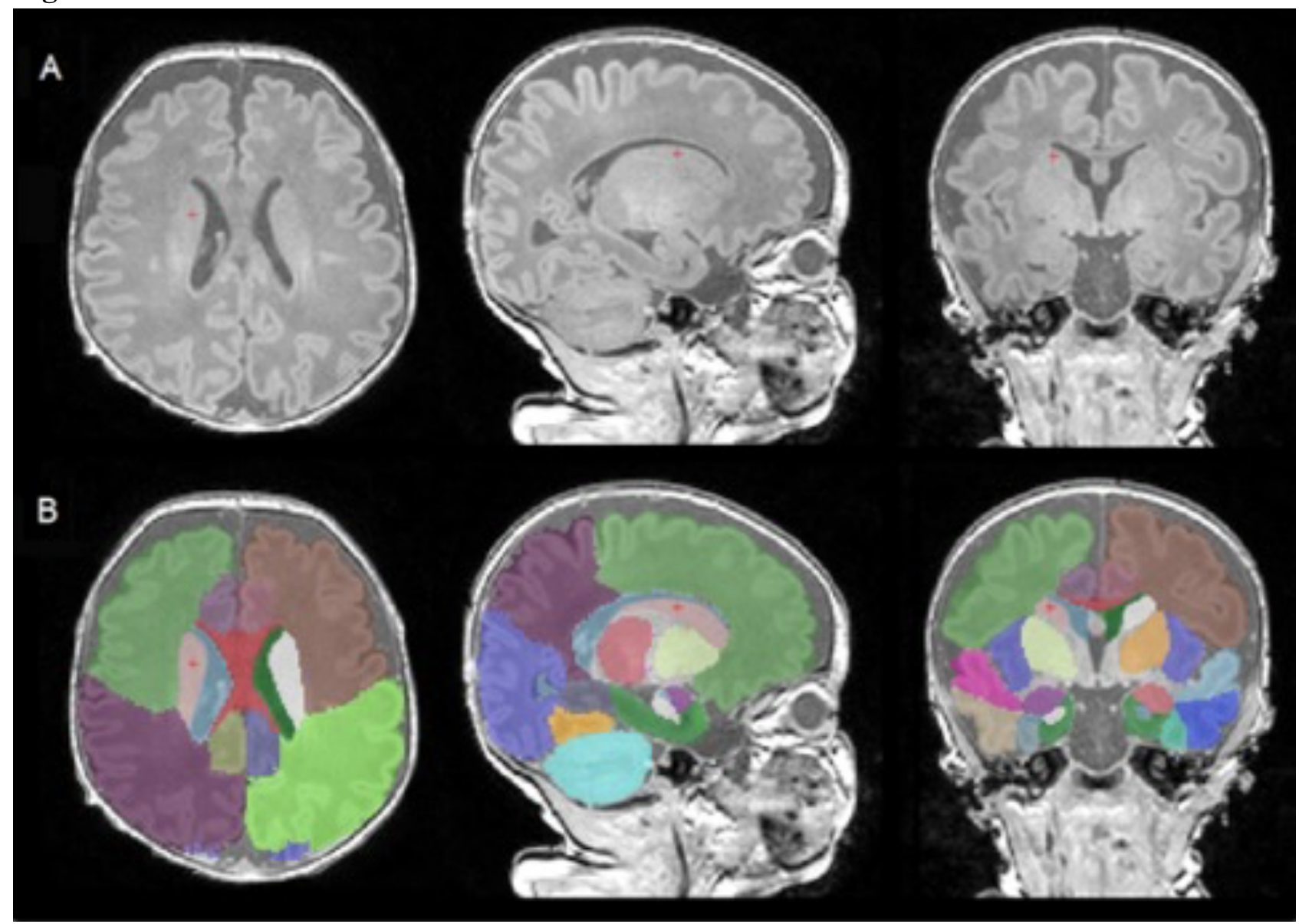

Figure 1. Neonatal (A) T1-weighted image and (B) automatic segmentation based on ALBERT atlas (from left to right: axial, sagittal and coronal views). 


\section{Figure 2}

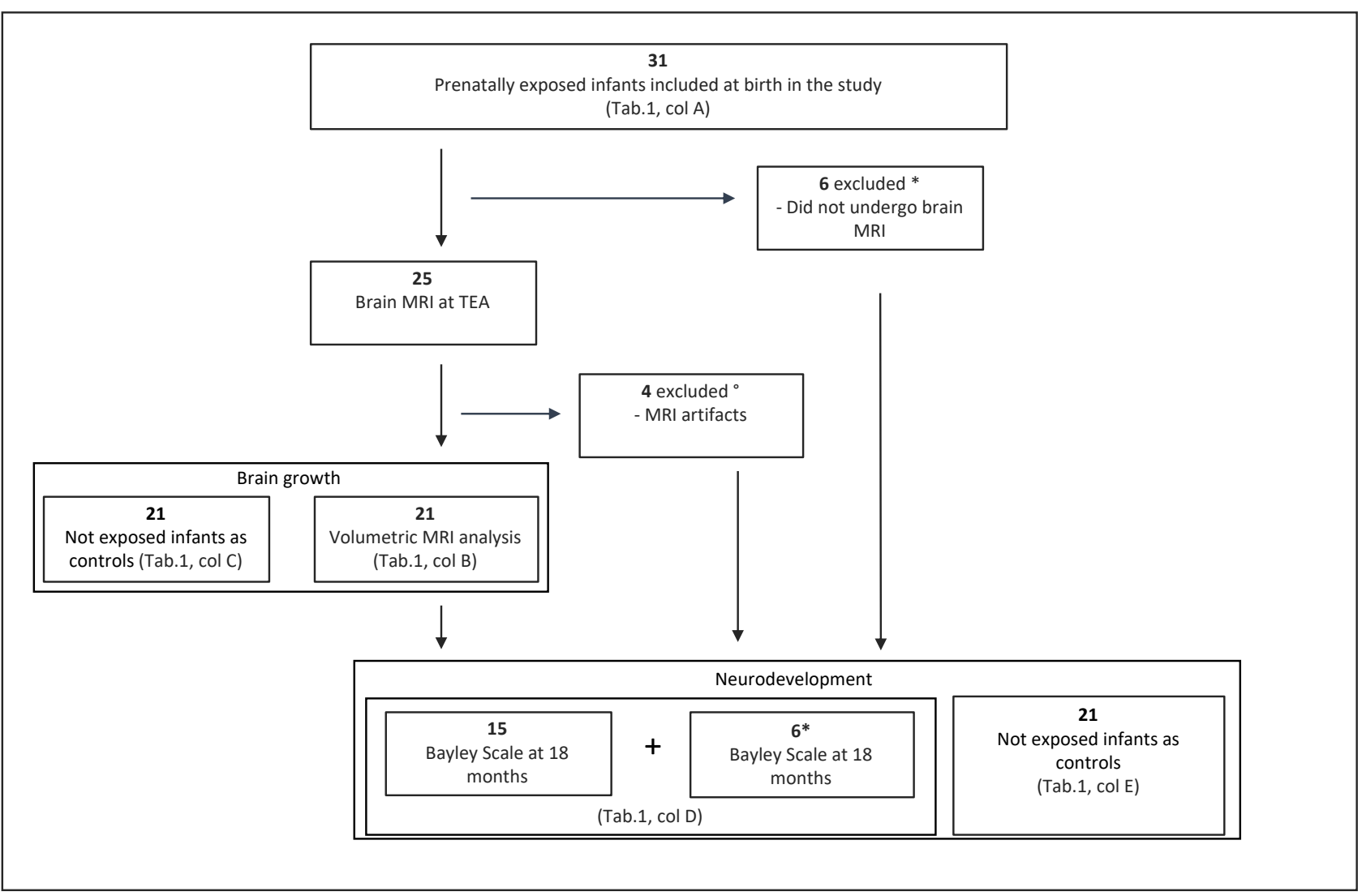

Figure 2. Flow chart of the Study Design and Recruitment. Thirty-one newborns were enrolled in the prenatally exposed group between 2012 and 2017 including 25 newborns who underwent brain MRI at term post menstrual age. Four infants were excluded from the MRI study due to motion artifacts in the MR images, which resulted in 21 MRI scans suitable for volumetric analysis. Twentyone of the 31 prenatally exposed children were assessed at 18 months of age by the Bayley test: 9 children were younger than 18 months, and one child was lost at follow-up. Fifteen prenatally exposed children underwent both brain MRI at term post menstrual age and neurodevelopmental assessment at 18 months * Four of 6 infants excluded from the MRI study because they did not undergo brain MRI were assessed at 18 months using the Bayley scales, while ${ }^{\circ}$ among the 4 infants excluded from the MRI study because of motion artifacts, 2 infants had Bayley Scales performed at 18 months. In brackets the indication regarding the characteristics of the population reported in table 1. 
Figure 3

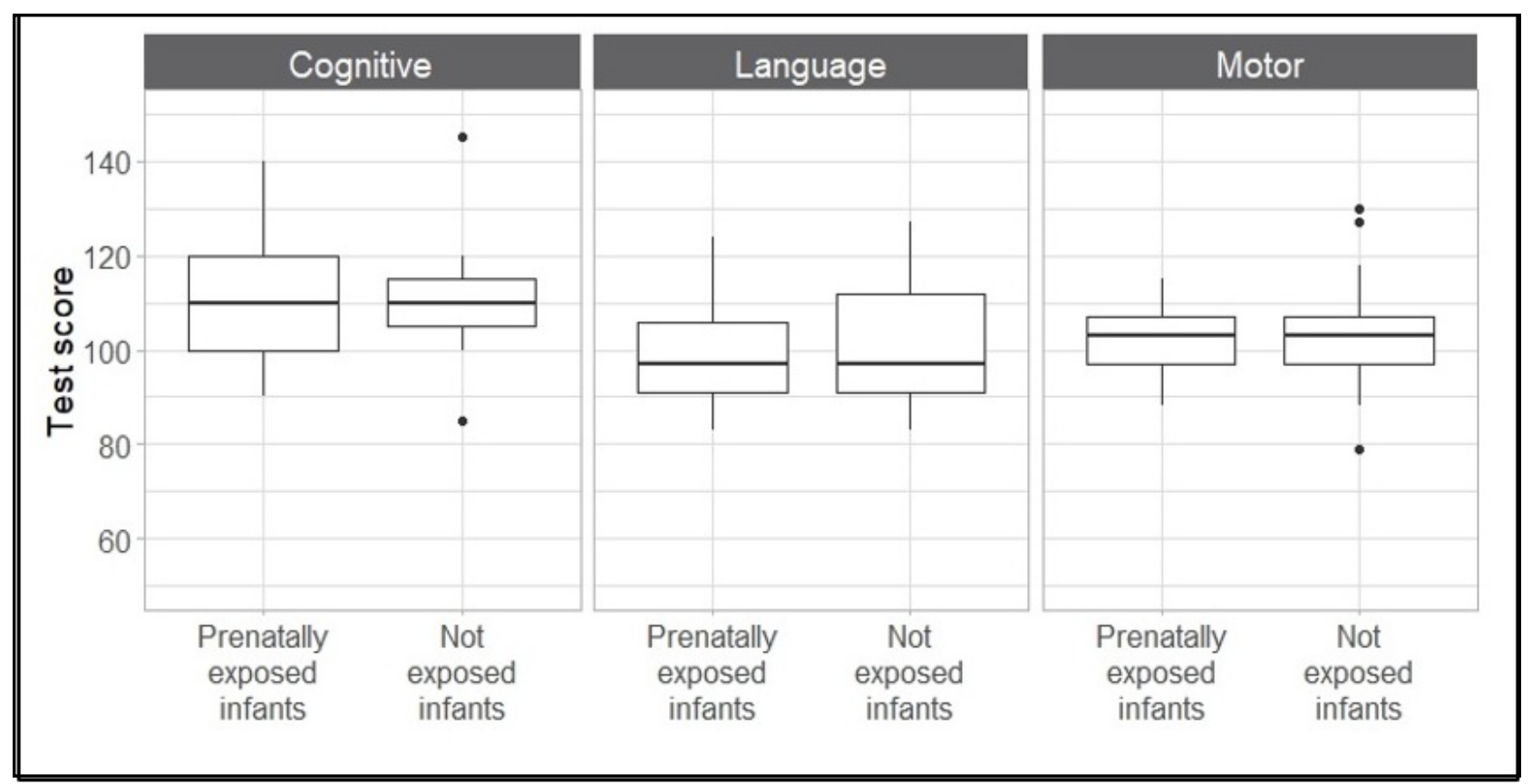

Figure 3. Neurodevelopment outcome at 18 months. Box plot shows the median score (solid horizontal line) and interquartile range (IQR = white box) for cognitive, motor and language scores in prenatally exposed and not exposed infants. Whiskers are extended to the most extreme data point that is no more than 1.5 $\times$ IQR away from the box. Data not included between the whiskers are plotted as outliers with a dot. Bayley scores of $10 / 21$ prenatally exposed infants were previously published [4]. 